\title{
Detection of ionizing radiation generated by electrical discharges in the air using sphere-sphere system
}

\author{
Eukasz Nagi ${ }^{1, *}$ \\ ${ }^{1}$ Opole University of Technology, Prószkowska 76, 45-758 Opole, Poland
}

\begin{abstract}
The article presents the results of studies on the detection of ionizing radiation generated during electrical discharges. The obtained energy spectra for a system of sphere spark gaps, and the dependence of the obtained energies on the geometric arrangements of the detection devices and of the electrical discharge generating devices are presented. Additionally, the recorded signals from the scintillation detector, and the energy spectrum received for the examined system are also presented. It is significant that the field distribution related to the shape of the spark gaps is of key importance for the received measurement data. The completed studies for the sphere - shaped spark gaps are a part of studies on recording ionizing radiation generated by full and partial discharges in the air and in mineral oil.
\end{abstract}

\section{Introduction}

Both full and partial discharges are a significant threat not only for human health and life, but also for electrical supply security. Nowadays, effective diagnostics of power equipment and networks is one of the most important tools for safe work and for ensuring a reliant power system, without the risk of e.g. uncontrolled electric power shortages. Electrical discharge detection is the main subject of the studies at the Institute of Electric Power Engineering and Renewable Energy (IEIEO). Laboratory experiments precede the application of innovative methods in the industry. Many methods of partial discharge detection developed at IEIEO have a practical application. The problem of the occurrence of electrical discharges and their effect on high voltage technology have resulted in developing numerous methods to detect these physical phenomena not only at the Opole University of Technology. The main reason for the world-wide works on this issue is that the sooner discharges are detected the faster the hazards can be reduced. On the contrary, the reduction of energy losses and economic losses is important for the development of technology, science, and the society's quality of life, therefore diagnostics of electrical engineering devices and facilities is very crucial. The existing methods can be divided into invasive and non-invasive methods. The invasive methods use a direct connection to the facilities being tested, and thus partial discharge currents can be detected with great precision. The non-invasive methods offer lower accuracy, but faster and more comfortable traceability of PD (partial discharges). At present, power network and device diagnostics are based on several electrical discharge detection methods. These are methods for detecting mostly PD that precede failures. The most effective methods include UHF [1-3], the acoustic emission method (EA) [4-6], ], the optical method [7-10]. Another result of the studies on electrical discharges is defining the power balance of the phenomenon. This would require determining energies coming from each type of energy that is released during a discharge. From light energy through heat, sound, to high frequency electromagnetic radiation. Electrical discharges generate ionizing radiation $[11,12]$ to, both in natural atmospheric discharges [13-15] and in laboratories [1618]. Studies on partial discharges in a laboratory proved the existence of such radiation also at a voltage between the electrodes not higher than $120 \mathrm{kV}[19,20]$. When preparing effective ionizing radiation detection methods on actual facilities, the energy balance of an electrical discharge phenomenon can be developed. The article presents the results obtained in the course of laboratory tests on a model spark gap.

\section{Measurement procedure and results}

A system that consists of the following three systems has been designed to generate and to measure highlyenergetic radiation:

-a system generating electrical discharges,

-a system to control the measurement desk, -a system to measure highly-energetic radiation.

The first part is comprised of a set of spark gaps powered with high voltage from a transformer, and the third part of a scintillation detector placed at the negative electrode or at a certain distance from this electrode. The detector is fitted on an arm that can be positioned at any place in the space with a precision of down to $0.1 \mathrm{~mm}$. During the measurement, the voltage was increased until the system's breakdown.

The tests with the sphere gap system with a sphere diameter of $20 \mathrm{~mm}$ were performed for 10 various

*Corresponding author: 1.nagi@po.opole.pl 
distances between the electrodes. For the research 172 measurements were performed to take a dataset. Fig. 1 characterizes the dependence between the maximum recorded energy and the distance between the spark gaps. With the increase in the distance, and hence the increase in the system's breakdown voltage, the maximum recorded ionizing radiation energy increases until it reaches the plateau for a given system. Figs. 2 - 4 present average scintillation energy in this system. Each diagram presents different models that match the fitting. A similar tendency can here again be observed, when along with an increase in the breakdown voltage (caused by an increase in the distance between the spark gaps) the maximum energy recorded by the detector increases. The presented models adjusted to the obtained data have high $\mathrm{R}^{2}$ coefficients, the best one is for the exponential model. Figs. 5 and 6 show the sum of scintillation energy in this system. Each chart reflects different models that match the fitting. As in previous characteristics concerning the maximum and the average energy recorded in the tests, it can be observed here that, along with an increase in the distance between the spark gaps, the energy recorded by the detector increases. The linear model is the best fitting for the total energy measured.

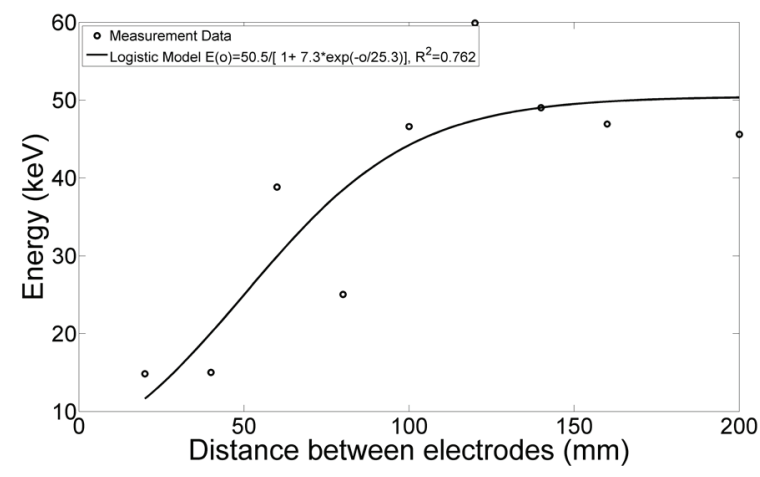

Fig. 1. The dependence between the maximum scintillation energy and the distance between the electrodes in a sphere gap system with a sphere diameter of $20 \mathrm{~mm}$ in a test performed in the air.

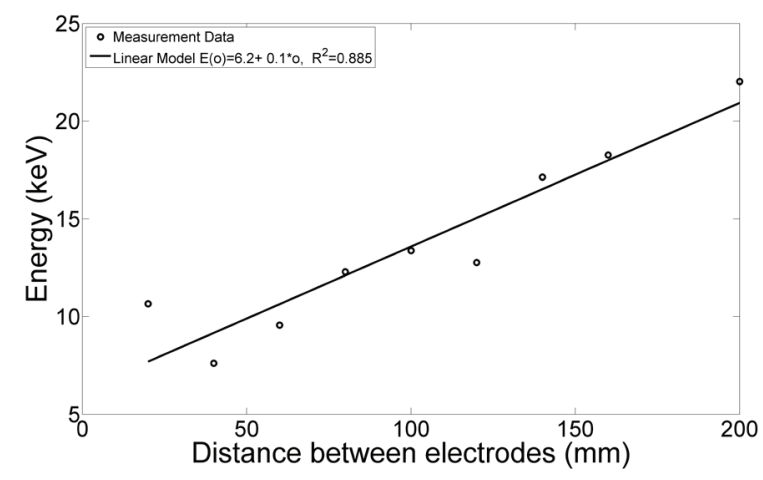

Fig. 2. The dependence between the average scintillation energy and the distance between the electrodes in the sphere gap system with a sphere diameter of $20 \mathrm{~mm}$ in a test performed in the air.

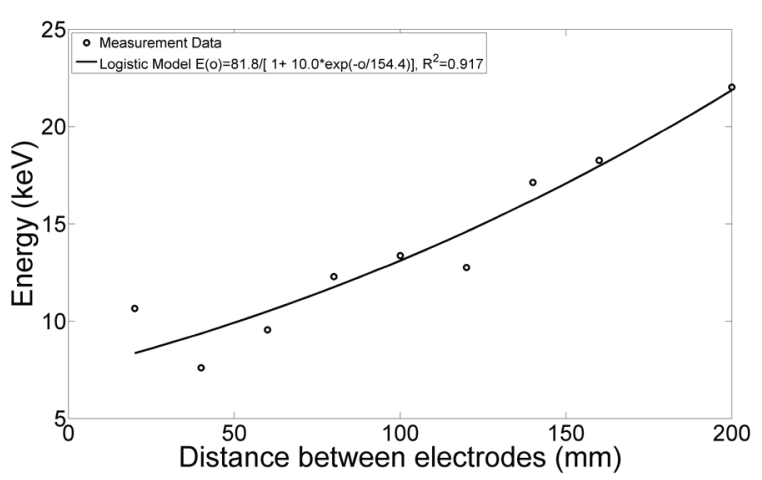

Fig. 3. The dependence between the average scintillation energy and the distance between the electrodes in the sphere gap system with a sphere diameter of 20 .

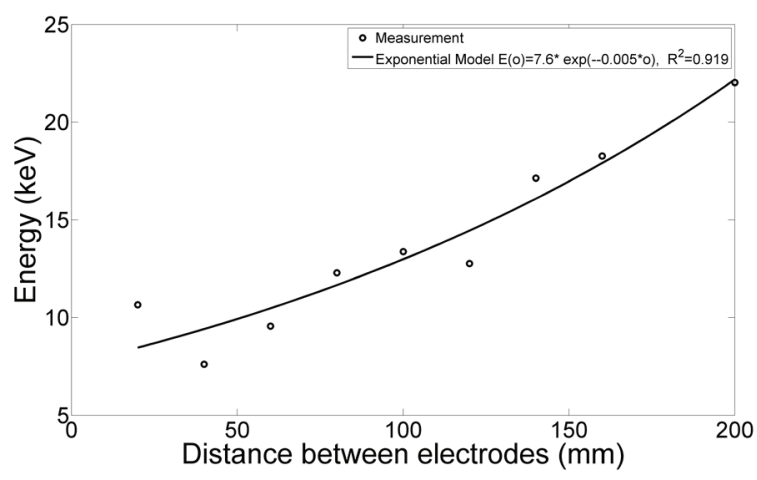

Fig. 4. The dependence between the average scintillation energy and the distance between the electrodes in the sphere gap system with a sphere diameter of 20 .

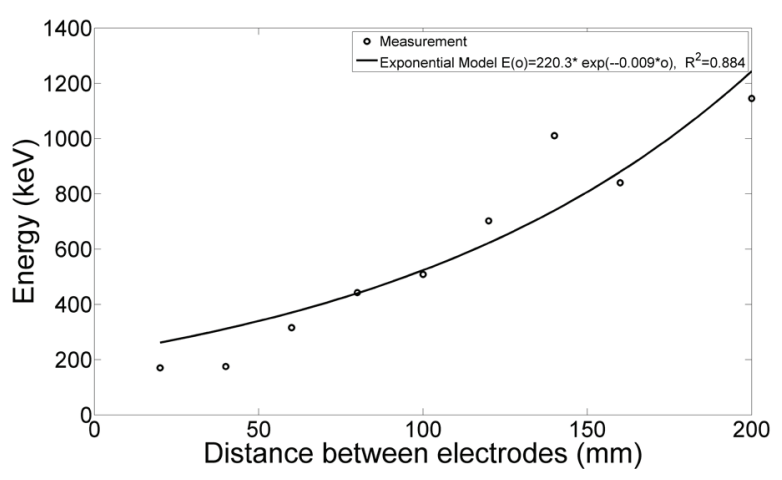

Fig. 5. The sum of the scintillation energy depending on the distance between the electrodes in the sphere gap system with a sphere diameter of 20 .

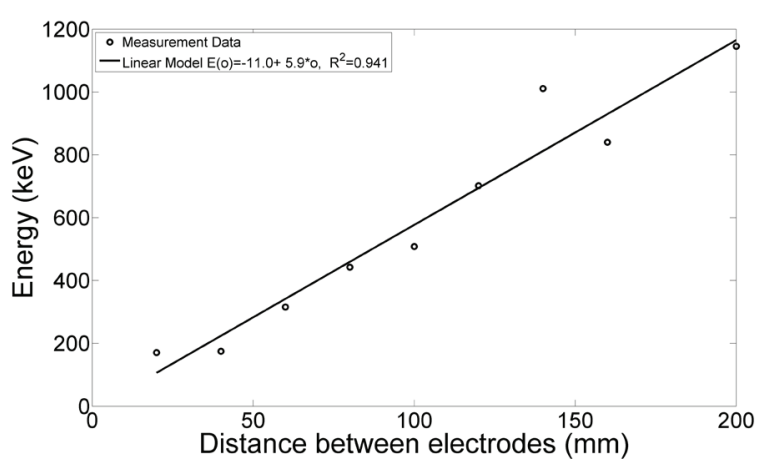

Fig. 6. The sum of the scintillation energy depending on the distance between the electrodes in the sphere gap system with a sphere diameter of $20 \mathrm{~mm}$. 
Fig. 7 presents the energy spectrum for the selected distances between the electrodes, while Fig. 8 shows the energy spectrum recorded in this electrode arrangement. With an increase in the distance between the electrodes, the recorded spectrum shifts towards higher energies, also, the total count number is higher for a greater distance between the spark gaps. The energy spectrum shows that for this test system, the highest energies recorded occur at higher voltage, and the highest count number in one energy interval falls between 60-120 mm of the distance between the electrodes.

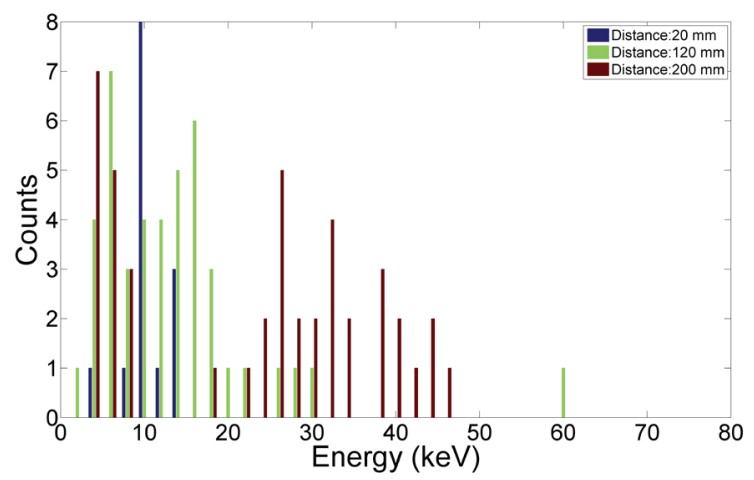

Fig. 7. The energy spectrum for the selected distances between the electrodes in the sphere gap system with a sphere diameter of $20 \mathrm{~mm}$ in a test performed in the air.

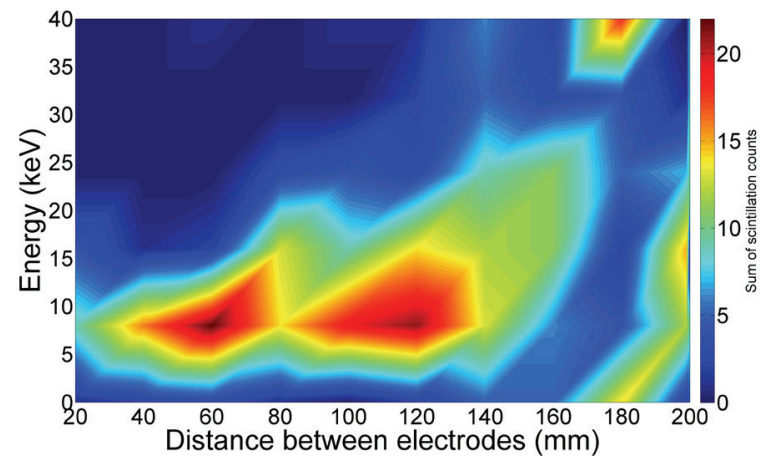

Fig. 8. The scintillation energy spectrum in the sphere gap system with a sphere diameter of $20 \mathrm{~mm}$ in a test performed in the air.

During the measurements, the voltage, the breakdown current, and the shape and number of scintillating bolts at the time of a breakdown in the system were recorded at the same time. Sample partial data registered in the test are presented in Figs. 9 and 10. They reflect the recorded scintillation peak, and the signal with noise and interference removed, respectively. Figs. 11 and 12 present exemplary scintillation models for a $\phi 20 \mathrm{~mm}$ sphere system in the air. Fig. 13 shows the time courses of the signals from the synchronized measuring voltage and current intensity systems, and from the ionizing radiation measuring system.

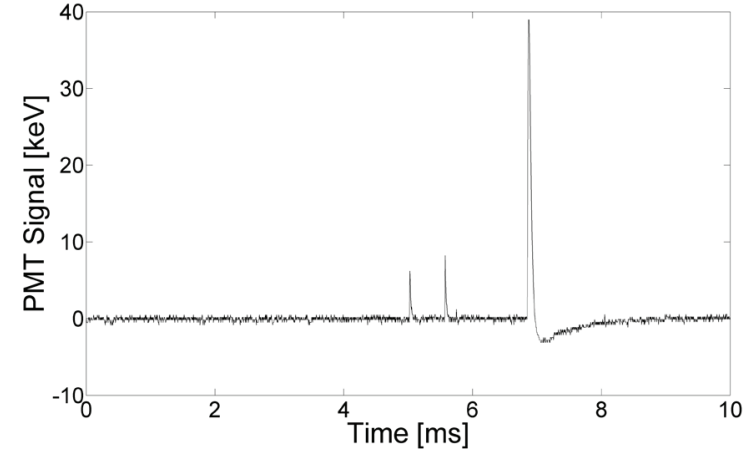

Fig. 9. Sample scintillation registered for the system. The distance between the $60 \mathrm{~mm}$ electrodes. The distance between the detector and the negative $1 \mathrm{~mm}$ electrode.

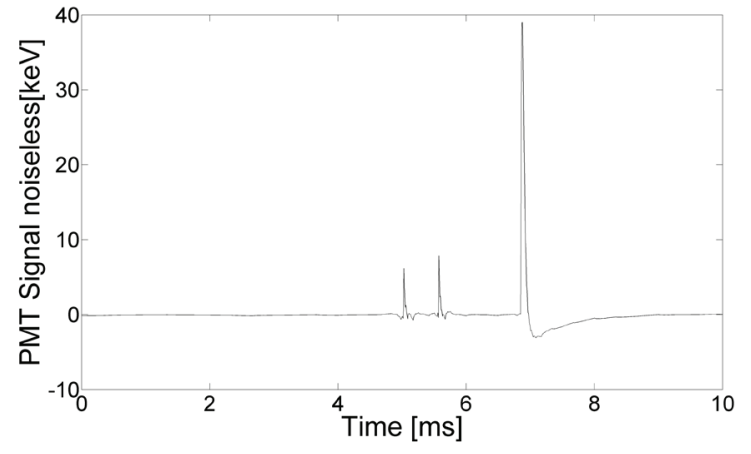

Fig. 10. Sample scintillation from figure 9 with noise and interference removed.

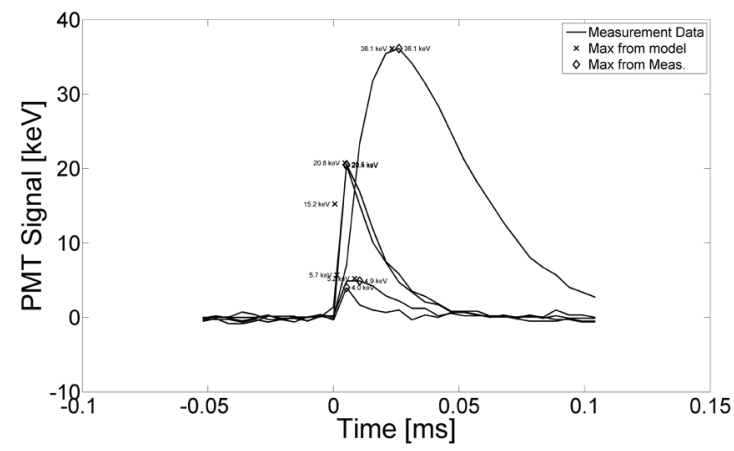

Fig. 11 Different mathematical models for a sample scintillation in the sphere gap system with a sphere diameter of $20 \mathrm{~mm}$ in a test performed in the air.

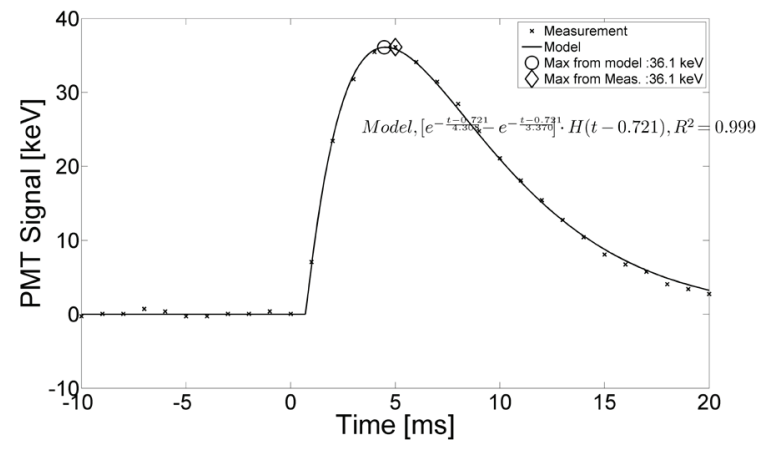

Fig. 12. The best fitted model for a sample scintillation in the sphere gap system with a sphere diameter of $20 \mathrm{~mm}$ in a test performed in the air. 

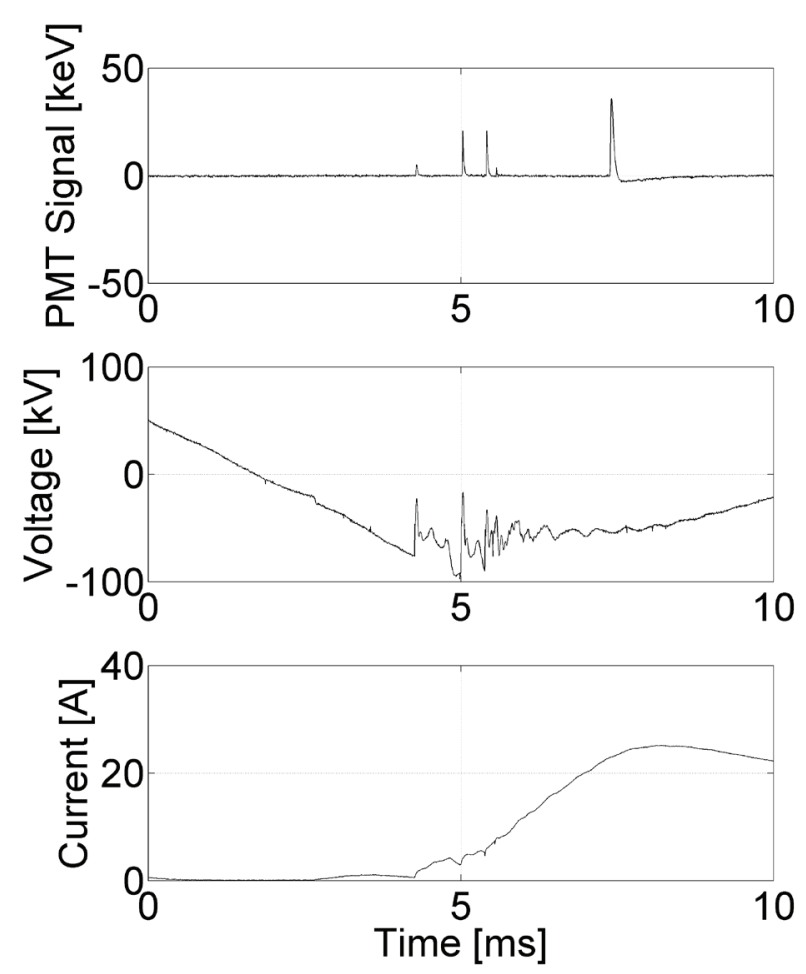

Fig. 13. The time courses of the signals from the multiplier photo-tube, and of the current and voltage signal on the primary winding in the transformer at breakdown.

\section{Conclusions}

The test in the sphere-sphere gap system demonstrated a high dependence of the data recorded on the distances between the electrodes. The maximum scintillation energy, the total energy in the measurement, and the average energy per scintillation in the measurement increase along with the increases in the distance between the spark gaps.

An increase in the distance increases the breakdown voltage, which, in turn, may result in an increase in the ionization energy of air molecules, and further to higher bremsstrahlung energies when excited molecules return to their primary state. The energy spectrum in this system suggests that the greatest number of scintillations is recorded at a distance between the electrodes equal to $120 \mathrm{~mm}$. An increase in the distance of more than $120 \mathrm{~mm}$ lowers the count of recorded scintillations, and the energy of individual excitations increases. Along with an increase in the distance, the radiation spectrum shifts towards higher energies, reducing the number of recorded scintillations at the same time.

\footnotetext{
ACKNOWLEDGEMENT

The work was co-financed from funds of the National Science Centre (NCN) as part of the PRELUDIUM programme, project No. 2014/15/N/ST8/03680
}

\section{References}

1. W. Gao, D. Ding, W. Liu, X. Huang, IEEE Trans. Power Deliv. 29, 38-47 (2014)

2. M. Kunicki, A. Cichoń, Meas. Autom. Monit. 61, 12-15 (2015)

3. M. Siegel, M. Beltle, S. Tenbohlen, IEEE Trans. Dielectr. Electr. Insul. 23, 1580-1588 (2016)

4. A. Hekmati, Int. J. Electr. Power Energy Syst. 77, 250-255 (2016)

5. D. Wotzka, T. Boczar, D. Zmarzly, Acta Phys. Pol. A - Opt. Acoust. Methods Sci. Technol. 116, 428-431 (2009)

6. M. Kunicki, A. Cichoń, S. Borucki, Arch. Acoust. 41, 265-276 (2016)

7. T. Boczar, D. Zmarzly, P. Fracz, IEEE 11th Int. Conf. Prop. Appl. Dielectr. Mater. 2015 740-743 (2015). doi:10.1109/ICPADM.2015.7295378

8. P. Frạcz, T. Boczar, S. Borucki, A. Cichoń, D. Zmarzły, Acta Phys. Pol. A 122, 814-817 (2012)

9. P. Fracz, IEEE Trans. Dielectr. Electr. Insul. 20, 1909-1914 (2013)

10. S. Biswas, C. Koley, B. Chatterjee, S. Chakravorti, IEEE Trans. Dielectr. Electr. Insul. 19, 18-28 (2012)

11. P. O. Kochkin, C. V Nguyen, a P. J. van Deursen, U. Ebert, J. Phys. D. Appl. Phys. 45, 425202 (2012)

12. P. O. Kochkin, A. P. J. van Deursen, U. Ebert, J. Phys. D. Appl. Phys. 48, 25205 (2015)

13. J. R. Dwyer et al., J. Geophys. Res. Sp. Phys. 117, n/a-n/a (2012)

14. J. R. Dwyer, J. Geophys. Res. 113, D10103 (2008)

15. P. Kochkin, A. P. J. van Deursen, A. de Boer, M. Bardet, J.-F. Boissin, J. Phys. D. Appl. Phys. 48, 425202 (2015)

16. J. R. Dwyer et al., J. Geophys. Res. 113, D23207 (2008)

17. J. R. Dwyer et al., Geophys. Res. Lett. 32, 1-4 (2005)

18. P. O. Kochkin, a P. J. van Deursen, U. Ebert, A. Van Deursen, U. Ebert, J. Phys. D. Appl. Phys. 47, 145203 (2014)

19. D. Zmarzly, L. Nagi, S. Borucki, T. Boczar, Acta Phys. Pol. A 125, 1377-1379 (2014)

20. Ł. Nagi, D. Zmarzły, T. Boczar, P. Frącz, IEEE Trans. Dielectr. Electr. Insul. 23, 2036-2041 (2016) 